\title{
Levels of biological markers of nitric oxide in serum of patients with squamous cell carcinoma of the oral cavity
}

\author{
Wioletta Ratajczak-Wrona ${ }^{1}$, Ewa Jablonska ${ }^{1}$, Bozena Antonowicz ${ }^{2}$, Dorota Dziemianczyk ${ }^{3}$ and Stanislawa \\ Zyta Grabowska ${ }^{3}$
}

The aim of the study was a determination of the levels of nitric oxide (NO) and its biological markers such as malonyldialdehyde (MDA) and nitrotyrosine in the serum of patients with squamous cell carcinoma (SCC) of the oral cavity and identification of the relationships between $\mathrm{NO}$ and those markers. These studies were performed on patients with SCC of the oral cavity before and after treatment. Griess reaction was used for the estimation of the total concentration of NO in serum. The nitrotyrosine level in serum was assessed with an enzyme-linked immunosorbent assay (ELISA) kit, and MDA level using a spectrophotometric assay. Higher concentrations of NO in blood serum were determined in patients with stage IV of the disease before treatment in comparison to the control group and patients with stages II and III of the disease. Moreover, higher concentrations of MDA and nitrotyrosine were determined in the serum of patients in all stages of the disease in comparison to healthy people. After treatment, lower concentrations of NO in the serum of patients with stage IV of the disease were observed in comparison to the amounts obtained prior to treatment. In addition, lower levels of nitrotyrosine in the serum of patients with all stages of the disease were recorded, whereas higher concentrations of MDA were determined in these patients in comparison to results obtained before treatment. The compounds formed with the contribution of NO, such as MDA and nitrotyrosine, may lead to cancer progression in patients with SCC of the oral cavity, and contribute to formation of resistance to therapy in these patients as well. Moreover, the lack of a relationship between concentrations of NO and MDA, and between NO and nitrotyrosine in serum suggests that the process of lipid peroxidation and nitration in patients with SCC does not just depend on NO. International Journal of Oral Science (2013) 5, 141-145; doi:10.1038/ijos.2013.59; published online 23 August 2013

Keywords: malonyldialdehyde; nitric oxide; nitrotyrosine; squamous cell carcinoma of the oral cavity

\section{INTRODUCTION}

Over $90 \%$ of head and neck cancers are squamous cell carcinomas (SCCs), which are the most common malignant cancer of the oral cavity. Each year, around 480000 new cases of oral cancer are diagnosed worldwide. In addition, simultaneous or progressive development of another tumor occurs in around $20 \%$ of patients. ${ }^{1-2}$

Development of SCC is conditioned by both external (cigarette smoke, mechanical injuries, viral and fungal infections) and internal (genetic and immunological defects) factors. Reactive oxygen and nitric species, including nitric oxide (NO), are also included in these factors. ${ }^{1,3}$

The results from many clinical and experimental studies have demonstrated that the role of $\mathrm{NO}$ in the carcinogenic process depends on compound concentration. Low levels of NO can promote tumor development, whereas its high concentration acts cytotoxically on tumor cells. ${ }^{4}$ This has been confirmed by the studies of Ohashi et $a l^{5}$ and Sumitani et al. ${ }^{6}$ who have demonstrated apoptotic changes in tumor cells in the presence of an NO donor. They have suggested that NO can result in regression of cancer through inhibition of DNA replication and prevention of mitochondrial respiration in tumor cells. Especially interesting from the clinical point of view of oral cancers are the data presented by Shang et al. ${ }^{7}$ who demonstrated that a high concentration of NO is an important inhibitory factor for the growth of oral SCC through the induction of apoptosis.

On the other hand, the procarcinogenic role of $\mathrm{NO}$ was also identified. Some earlier publications have demonstrated that $\mathrm{NO}$ at both low and high concentrations may promote carcinogenesis through the activation of the transcription hypoxia-inducible factor 1 (HIF-1). ${ }^{8}$ This process is time- and cell type-dependent. HIF-1 is subjected to destabilization and proteasomal degradation in normoxic conditions, while in hypoxia conditions, its overexpression is observed. An overexpression of HIF-1 transcription factor, as one of the mechanisms of cancer cells adaptation to the conditions of oxygen deficiency, is connected inter alia to changes in intercellular metabolism. ${ }^{9}$ It has been observed that high HIF-1 expression in SCC cells of the oral cavity is correlated with their increased resistance to radio- and chemotherapy that may result in an increase in primary cancer tumor and additionally favor secondary foci formation. ${ }^{10}$

One consequence of an increased concentration of NO may be its direct effect on the building components of cells in an organism. ${ }^{11}$

It has been established that NO plays an essential role in the process of lipid peroxidation. This is a free radical chain process in which there

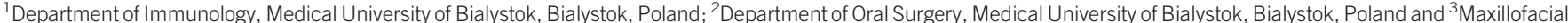
and Plastic Surgery Clinic, Medical University of Bialystok, Bialystok, Poland

Correspondence: Dr W Ratajczak-Wrona, Department of Immunology, Medical University of Bialystok, Waszyngtona 15A, Bialystok 15-269, Poland

E-mail: rwioletta@umb.edu.pl

Received 22 December 2012; accepted 26 June 2013 
is oxidation of polyunsaturated fatty acids or unsaturated fatty acid moieties, included in the composition of phospholipids - the main building component of cell membranes. Unlike proteins and nucleic acids, the process of lipid peroxidation is characterized by a chain reaction that results in the generation of a large number of peroxides of unsaturated fatty acids or other lipids. ${ }^{11}$

One of the final products of lipid peroxidation is malonyldialdehyde (MDA) that contains two reactive aldehyde groups which can react with two different molecules $\left(\mathrm{R}_{1}-\mathrm{NH}_{2}\right.$ and $\left.\mathrm{R}_{2}-\mathrm{NH}_{2}\right)$ and can 'sew' them into products with a characteristic structure $\left(\mathrm{R}_{1}-\mathrm{N}=\mathrm{CH}-\right.$ $\mathrm{CH}=\mathrm{NH}-\mathrm{R}_{2}$ ) called Schiff bases $\mathrm{N}, \mathrm{N}^{\prime}$-amino imino-propene. ${ }^{11}$ It has been proven that aldehydes formed as a result of lipid peroxidation are less reactive than free radicals and thereby can diffuse to significant distances in cells; therefore, they play the role of 'the secondary mediators' of damage caused by reactive oxygen and nitrogen species. Aldehydes react mostly with thiol and amine groups of proteins, lipids, amino sugars and nitrogenous bases of nucleic acids. They modify physical properties of cell membranes by increasing their permeability in respect of $\mathrm{H}^{+}$ions and other polar substances. ${ }^{12-13}$ This causes changes in electric potentials on both sides of the membrane, resulting in loss of integration of the intracellular membranes and the plasmatic membrane and inhibition of activity of membrane enzymes and carrier proteins. ${ }^{14}$

$\mathrm{NO}$ is also included among the main factors responsible for nitrification of the phenol groups of tyrosine in tissues and blood proteins. Nitrotyrosine, which is formed in this process, can cause a loss of the biological function of blood proteins and result in pathological changes. Nitrotyrosine concentration can be a helpful marker for the evaluation of NO action under in vivo conditions. Moreover, nitrotyrosine, because of its longer half-life, may be a better indicator of the increased production of NO than metabolites of NO. ${ }^{15-16}$

The aim of the study was the determination of the total concentration of NO, MDA and nitrotyrosine in the serum of patients with SCC of the oral cavity and identification of the relationship between these parameters, which could extend knowledge about the role of NO and markers of NO activity during the pathogenesis of cancer in the studied group of patients.

\section{MATERIAL AND METHODS}

We examined 24 patients with SCC of the oral cavity treated in the Maxillofacial and Plastic Surgery Clinic, Medical University of Bialystok. Assays were performed before the treatment and 3 weeks after surgical removal of the tumor mass. Study results were analyzed taking into account a clinical stage of the disease according to tumour node metastasis staging system (TNM classification system) (Table 1). ${ }^{17}$ Characteristic of patients was presented in the Table 1. Patients for one week after surgery received Morphini Sulfas (10 mg every $6 \mathrm{~h}$ for 3 days; Polfa Tarchomin S.A., Warsaw, Poland), paracetamol (1 $000 \mathrm{mg}$ every $12 \mathrm{~h}$ for 7 days, Perfalgan; Bristol-Myers Squibb, Uxbridge, UK) for postoperative pain and antibiotics cephalosporin ( 1 g every 12 h for 7 days, Tarfazolin; Polfa Tarchomin S.A., Warsaw, Poland). All females and males participated in the study were cigarette smokers for over 10 years. Control subjects $(n=15)$ were non-smoking healthy volunteers aged from 30 to 60 years (mean \pm s.d.: $42.5 \pm 15.3$ years). None of the patients and control subject had concomitant diseases such as diabetes mellitus, liver disease or rheumatoid arthritis.

The study was approved by the Ethics Committee of the Medical University of Bialystok (R-I-003/87/2006).
Table 1 Clinical characteristic of patients with squamous cell carcinoma of the oral cavity

\begin{tabular}{lccc}
\hline \multirow{2}{*}{ Characteristics } & \multicolumn{3}{c}{ Number of patients in TNM classification } \\
\cline { 2 - 4 } & Stage II & Stage III & Stage IV \\
\hline Patients & 8 & 8 & 8 \\
Male/female & $7 / 1$ & $6 / 2$ & $8 /-$ \\
Mean age & 68 & 69 & 67 \\
Site of cancer & & & \\
$\quad$ Oral cavity fundus & $1 /-$ & $2 /-$ & $4 /-$ \\
$\quad$ Tongue & $1 /-$ & $1 / 1$ & $-/-$ \\
$\quad$ Tongue+oral cavity & $1 /-$ & $1 / 1$ & $-/-$ \\
$\quad$ undus & & & \\
$\quad$ Cheek mucosa & $1 / 1$ & $-/-$ & $1 /-$ \\
$\quad$ Inferior gingival & $1 /-$ & $1 /-$ & $2 /-$ \\
$\quad$ Lower lip & $2 /-$ & $1 /-$ & $1 /-$ \\
\hline
\end{tabular}

\section{Blood sampling}

Patients with SCC of the oral cavity and healthy control subjects were recruited into the study after obtaining their informed consent. Patients were not smoking cigarettes within $24 \mathrm{~h}$ prior to blood collection. Five milliliters of fasting blood was collected by venous arm puncture under aseptic conditions. Serum was obtained by centrifugation at $2000 \mathrm{~g}$ for $5 \mathrm{~min}$ of blood samples taken without anticoagulant. Serum was kept at $20{ }^{\circ} \mathrm{C}$ until the analysis date.

Determination of total $\mathrm{NO}\left(\mathrm{NO}_{3}{ }^{-} / \mathrm{NO}_{2}{ }^{-}\right)$concentration in serum Nitrite $\left(\mathrm{NO}_{2}{ }^{-}\right)$and nitrate $\left(\mathrm{NO}_{3}{ }^{-}\right)$are stable final products of $\mathrm{NO}$ metabolism and may be used as indirect markers of NO presence. Total NO concentration is commonly determined as a sum of nitrite and nitrate concentrations. NO concentration was determined using an indirect method based on measurement of nitrite concentration in serum according to Griess's reaction. ${ }^{18}$ In the samples analyzed, nitrate were reduced to nitrite in the presence of cadmium (SigmaAldrich, Steinheim, Germany), and then converted to nitric acid that gave a color reaction with Griess's reagent (Sigma-Aldrich, Stainheim, Germany). Nitrite concentrations were determined by spectrophotometric analysis at $540 \mathrm{~nm}$ (UVN-340 ASYS Hitech GmbH microplate reader; Biogenet, Eugendorf, Austria) with reference to a standard curve. NO products were expressed as $\mu$ moles.

\section{Determination of MDA concentration in serum}

The evaluation of MDA in serum was done by the method of Buege and Aust. ${ }^{18}$ The reaction is based on fusion of lipids oxidation products with thiobarbituric acid leading to creation of colored product. In the first step, $200 \mu \mathrm{L}$ of $50 \%$ trichloroacetic acid was added to $200 \mu \mathrm{L}$ of serum, and then mixed until precipitation of proteins and centrifugated at $5000 \mathrm{~g}$ for $5 \mathrm{~min}$. Following addition of $160 \mu \mathrm{L}$ of thiobarbituric acid to $160 \mu \mathrm{L}$ of supernatant collected after centrifugation, samples were incubated in a water bath at $90{ }^{\circ} \mathrm{C}$. After $30 \mathrm{~min}$, the colored product by the reaction of thiobarbituric acid with MDA was measured colorimetrically at $533 \mathrm{~nm}$ using DU SERIES 600 spectrophotometer (Beckman Coulter, Brea, CA, USA). The obtained results were expressed as nmoles.

\section{Determination of nitrotyrosine concentration in serum}

The nitrotyrosine level in serum was assessed by sandwich enzymelinked immunosorbent assay (ELISA) using a commercially available kit (Nitrotyrosine ELISA kit; Hycult Biotechnology b.v., PB Uden, The Netherlands). Before performing the assay, serum was diluted 10 times with dilution buffer (protein-stabilized phosphate-buffered saline, 
containing 2-chloroacetamide as preservative). Nitrotyrosine standards or serum $(100 \mu \mathrm{L})$ were pipetted into an antibody-coated 96well plate and incubated at room temperature for $1 \mathrm{~h}$. The wells were then washed four times with wash buffer (containing Tween-20), $100 \mu \mathrm{L}$ of anti-human nitrotyrosine antibody was added and the samples were again incubated for $1 \mathrm{~h}$ at room temperature. The plate was washed four times, $100 \mu \mathrm{L}$ of streptavidin-peroxidase conjugated was then applied for $1 \mathrm{~h}$ at room temperature. After a final washing, $100 \mu \mathrm{L}$ of tetramethylbenzidine substrate was added and allowed to develop for 20-30 min in the dark at room temperature. After stopping the reaction with stop solution (containing citric acid, $2.0 \mathrm{mmol} \cdot \mathrm{L}^{-1}$ ) $(100 \mu \mathrm{L})$, absorbance was read at $450 \mathrm{~nm}$ with a UVN-340 ASYS Hitech $\mathrm{GmbH}$ microplate reader (Biogenet, Eugendorf, Austria). The sample concentration was calculated from the standard curve. The obtained results were expressed as nmoles.

\section{Statistical evaluation}

The data obtained were subjected to statistical analysis using Microsoft Excel calculation sheet and STATISTICA version 9.1 (StatSoft Inc., Tulsa, OK, USA). All data are presented as mean \pm s.d. Data distribution normality was determined using Kolmogorov-Smirnov test. Since the data were not normally distributed, for comparison of variations between assayed groups, Mann-Whitney $U$ non-parametric tests were applied to unrelated results. A statistical significance level of $P<0.05$ was assumed. A correlation analysis was undertaken using Pearson's rank correlation test.

\section{RESULTS}

Our findings on the assessed parameters in SCC patients and healthy control subjects are shown in Figures $1-3$ and Table 2.

Figure 1 shows the levels of serum total concentration of $\mathrm{NO}$ $\left(\mathrm{NO}_{3}{ }^{-} / \mathrm{NO}_{2}{ }^{-}\right)$in control subjects and SCC patients. In the serum of patients with stage IV of the disease before and after treatment, significantly higher values for total NO concentrations were observed in comparison to the control group. Total concentrations of NO in the serum of patients with stage IV of the disease before treatment were higher in comparison to the patients with stages II and III of the disease. After treatment, lower concentrations of total NO were observed in patients with stage IV of the disease in comparison to values obtained before treatment. No differences were observed in total NO levels in the serum of patients with stages II and III of the disease after treatment in comparison to values obtained before treatment and to the control group.

Figure 2 presents the concentration of MDA in the control subjects and SCC patients. Significantly higher values for MDA concentrations in serum of all patients before and after treatment in comparison to the control group were noted. In addition, higher concentrations of MDA were noted in serum of patients after treatment in comparison to values obtained before treatment regardless of tumor stage. Increasing concentrations of MDA in serum of patients before and after treatment following the stage of disease progression were observed. A significantly higher concentration of MDA was observed in serum of patients with stage IV of the disease before and after treatment in comparison to patients with stages II and III of the disease.

Figure 3 shows the concentration of serum of nitrotyrosine in the control subjects and SCC patients. Significantly higher values for nitrotyrosine concentrations in the serum of all patients before treatment were demonstrated in comparison to the control group. In addition, concentrations of nitrotyrosine in serum of patients before the treatment were observed to increase with the progression of the stage

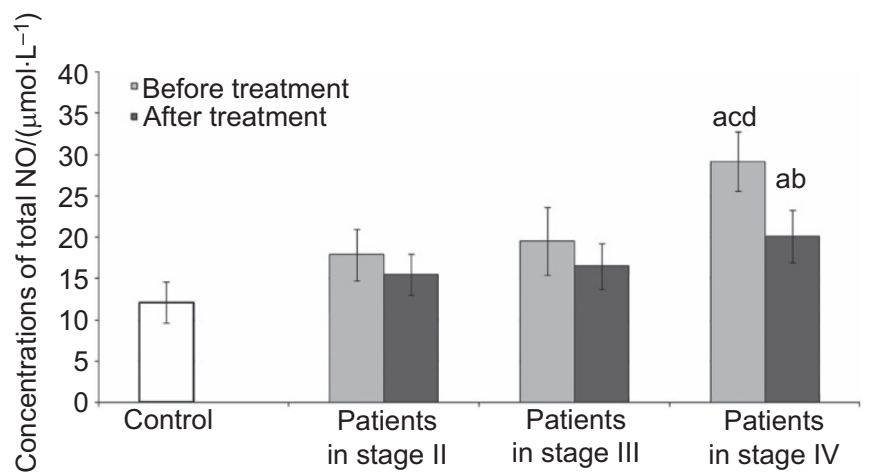

Figure 1 Concentrations of total NO in the serum of patients in different stage of disease. NO, nitric oxide. a, Statistical differences with control $(P<0.05)$; b, statistical differences between patients before and after treatment $(P<0.05)$; $\mathrm{c}$, statistical differences between patients in stage $\mathrm{II}$ and patients in stage IV $(P<0.05)$; d, statistical differences between patients in stage III and patients in stage IV $(P<0.05)$.

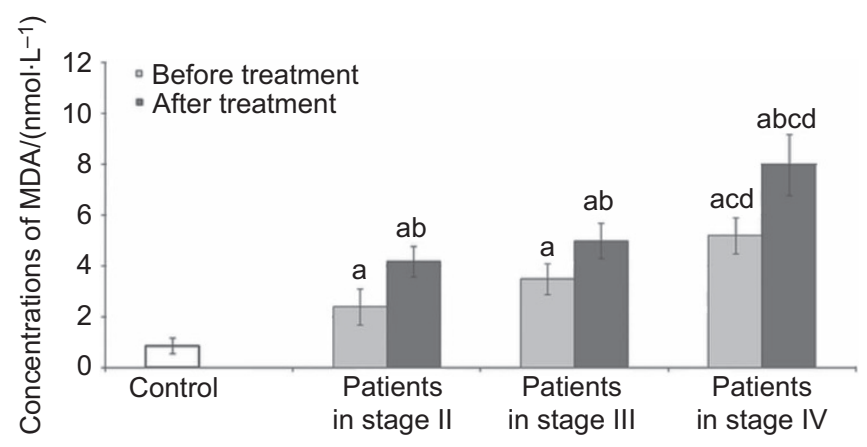

Figure 2 Concentrations of MDA in the serum of patients in different stage of disease. MDA, malonyldialdehyde. a, Statistical differences with control $(P<0.05)$; b, statistical differences between patients before and after treatment $(P<0.05)$; $c$, statistical differences between patients in stage II and patients in stage IV $(P<0.05)$; d, statistical differences between patients in stage III and patients in stage IV $(P<0.05)$.

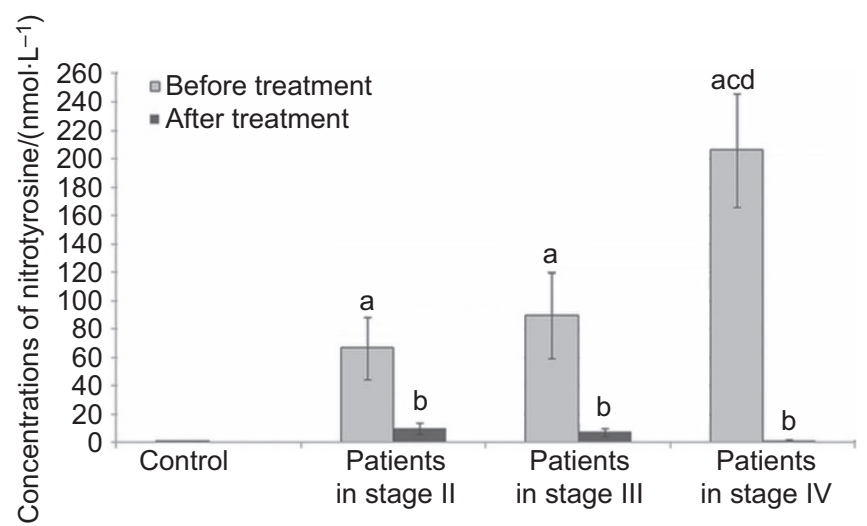

Figure 3 Concentrations of nitrotyrosine in the serum of patients in different stage of disease. a, Statistical differences with control $(P<0.05)$; b, statistical differences between patients before and after treatment $(P<0.05)$; $\mathrm{c}$, statistical differences between patients in stage II and patients in stage IV $(P<0.05)$; d, statistical differences between patients in stage III and patients in stage IV $(P<0.05)$ 
Table 2 Pearson's rank correlation between MDA concentrations and nitrotyrosine levels in serum of patients in different stage of disease before treatment

TNM classification

\begin{tabular}{llll}
\cline { 2 - 4 } Compound & Patients in stage II & Patients in stage III & Patients in stage IV \\
\hline Nitrotyrosine & MDA & MDA & MDA \\
& $r=0.61, P<0.05$ & $r=0.68, P<0.05$ & $r=0.75, P<0.05$ \\
\hline
\end{tabular}

MDA, malonyldialdehyde.

of the disease. However, in serum of patients after treatment, significantly lower concentrations of nitrotyrosine were established in comparison to values obtained before the treatment.

Table 2 presents the Pearson's rank correlations analysis between the MDA and nitrotyrosine levels in serum SCC patients before treatment. The analysis of relationships between concentrations of the biological markers of NO in serum of patients proved a positive significant correlation between concentrations of MDA and nitrotyrosine in serum of all patients before treatment. However, no statistically significant correlations were observed between the studied parameters in serum of all the patients after the treatment (data not presented).

\section{DISCUSSION}

NO, which is produced by many cells including cells of the immune system, can affect the systemic action of many different organs and tissues, as well as tumor cells. ${ }^{3,19}$

The high concentrations of total NO observed in our own studies in the serum of patients with SCC of the oral cavity before treatment may play a role in elimination of tumor cells. There are data that indicate that high levels of NO result in apoptosis of cells through direct damage of DNA, inhibition of proteins and nucleic acid synthesis. ${ }^{3,20}$

High concentrations of NO in the serum of patients with SCC of the oral cavity in the advanced stage of the disease were also obtained by Beevi et al., ${ }^{21}$ Rasheed et al. ${ }^{22}$ and Gokul et al. ${ }^{23}$

One of the causes of high concentrations of total NO in the serum of patients in the advanced stage of the disease could be secretion of NO by cells of the immune system. Jablonska et al. ${ }^{24}$ demonstrated higher expression and concentration of inducible nitric oxide synthase (iNOS), the enzyme responsible for the generation of NO, in polymorphonuclear cells and peripheral blood mononuclear cells in those patients, whereas lower expression and concentration of iNOS in polymorphonuclear cells and peripheral blood mononuclear cells independent of the stage of the disease was recorded in patients after treatment. Similar observations concerning the increase in the expression and activity of iNOS in patients with SCC of the oral cavity in comparison with weak or no activity of iNOS in the mucous membrane of healthy people have been made by Gavilanes et al. ${ }^{25}$

Our own studies showed that lower concentrations of NO in the serum of patients after surgical removal of the tumor mass may result from decreased production of NO through polymorphonuclear cells and peripheral blood mononuclear cells, as well as suggest a role of tumor cells in the secretion of this molecule. ${ }^{26}$

On the other hand, Connelly et al. ${ }^{27}$ reported significantly higher expression of iNOS in oral squamous carcinoma tissue linking it with high levels of NO in its pathogenesis. Thomsen et al. ${ }^{28}$ have demonstrated that iNOS inhibition may reduce the growth of xenografted tumors in vivo. There are studies that report the effect of NO upon tumor growth of mutant p53 tumors by promoting endothelial mitogenesis. ${ }^{29}$ Increased production of NO by cells of the immune system and tumor cells, besides affecting various systems and organs, can also influence the process of neoangiogenesis. ${ }^{30}$ It has been demonstrated that NO modulates the release of vascular endothelial growth factor by tumor cells, as well as initiating activation of matrix metalloproteinases, enzymes influencing the degradation of basement membrane and other cellular components. ${ }^{31-33}$ Another mechanism by which NO might promote carcinogenesis is probably by modulating the production of prostaglandins. NO increases the production of prostaglandin E2 by enhancing the prostaglandin synthase which results in an increased leakage in tumor vasculature. ${ }^{34}$

In our own studies, higher concentrations of MDA in the serum of all patients with SCC of the oral cavity both before and after the treatment were observed, which suggests intensified lipid peroxidation in these patients.

A consequence of high concentrations of MDA in serum of these patients could be that morphological and functional changes of many cells may finally result in the dysfunction of individual organs. Participation of MDA in the inactivation of protein synthesis and processes of replication and transcription of DNA was identified. It was observed that MDA's reaction with nitrogenous bases of nucleic acids could have a mutagenic function. ${ }^{35}$

One of the causes of high concentrations of MDA in the serum of patients in the advanced stage of the disease in comparison to values obtained in patients during the early stages of the disease was the lowered total anti-oxidative ability of peripheral blood, whereby the peripheral blood was unable to prevent uncontrolled oxidation. ${ }^{21,36}$ This is confirmed by results obtained by Sabitha et al. ${ }^{37}$ in patients with SCC of the oral cavity with stage III of the disease before and after treatment with radiotherapy. They demonstrated a high level of MDA in blood plasma of these patients in comparison to the control group, with a simultaneous decrease in the activity of anti-oxidative enzymes in blood cells.

Similar results were obtained by Korde et al., ${ }^{38}$ who observed a high level of MDA and total NO in serum of SCC patients with clinical stage III/IV.

High MDA levels despite low serum NO concentrations in the serum of patients with stages II and III of the disease, can be explained by the fact that other compounds such as sulfur dioxide, hydroxyl radicals and radical cations exhibit lipid peroxidation potential. ${ }^{18}$

Moreover, high concentrations of total NO together with the higher concentrations of nitrotyrosine observed in our own study in patients with stage IV of the disease according to TNM classification confirm the role of this molecule in the nitration process in this group of patients.

Nitration of tyrosine is mostly an irreversible process that can result in the inhibition of tyrosine phosphorylation and increased sensitivity of proteins towards proteolytic enzymes. ${ }^{39}$

It has been demonstrated that due to its increased immunogenicity, nitrotyrosine modulates the inflammatory process. ${ }^{40}$ Increased concentration of nitrotyrosine in the serum of patients with SCC of the oral cavity can facilitate development of tumors. There are published data which indicate the critical role of inflammation in tumor promotion. $^{41}$

The relationships between concentrations of MDA and nitrotyrosine observed in the present study in the serum of patients with SCC before treatment may negatively affect the reaction of host-cancer cells, and lead to impairment of anticancer response.

In conclusion, the compounds formed with the contribution of NO, such as MDA and nitrotyrosine, may lead to cancer progression in patients with SCC of the oral cavity, and contribute to formation of resistance to therapy in these patients as well. Moreover, the lack of a relationship between concentrations of $\mathrm{NO}$ and MDA, and between 
$\mathrm{NO}$ and nitrotyrosine in serum suggests that the process of lipid peroxidation and nitration in patients with SCC does not just depend on NO.

Further studies evaluating the biological activity of NO could lead to the development of new therapeutic methods using selective donors of $\mathrm{NO}$ or inhibitors of NO synthase in patients with SCC of the oral cavity.

\section{ACKNOWLEDGEMENTS}

This work was funded by the Medical University of Bialystok, Poland (project no: 3-06429F). Publication of this manuscript is supported by Open Fund of State Key Laboratory of Oral Diseases, Sichuan University.

1 Chen AY, Myers JN. Cancer of the oral cavity. Dis Mon 2001; 47(7): 275-361.

2 Massano J, Regateiro FS, Januario G et al. Oral squamous cell carcinoma: review of prognostic and predictive factors. Oral Surg Oral Med Oral Pathol Oral Radiol Endod 2006; 102(1): 67-76.

3 Moncada S, Higgs EA. Molecular mechanisms and therapeutic strategies related to nitric oxide. FASEB J 1995; 9(13): 1319-1330.

4 Xu W, Liu LZ, Loizidou M et al. The role of nitric oxide in cancer. Cell Res 2002 12(5/6): 311-320.

5 Ohashi M, Iwase M, Nagumo M. Elevated production of salivary nitric oxide in oral mucosal diseases. J Oral Pathol Med 1999; 28(8): 355-359.

6 Sumitani K, Kamijo R, Nagumo M. Cytotoxic effect of sodium nitroprusside on cance cells: involvement of apoptosis and suppression of c-myc and c-myb proto-oncogene expression. Anticancer Res 1997; 17(2A): 865-871.

7 Shang ZJ, Li JR, Li ZB. Effects of exogenous nitric oxide on oral squamous cell carcinoma: an in vitro study. J Oral Maxillofac Surg 2002; 60(8): 905-911.

8 Sandau KB, Fandrey J, Brüne B. Accumulation of HIF- $1 \alpha$ under the influence of nitric oxide. Blood 2001; 97(4): 1009-1015.

9 Vaupel P. The role of hypoxia-induced factors in tumor progression. Oncologist 2004, 9(5): 10-17.

10 Koukourakis MI, Giatromanolaki A, Sivridis E et al. Hypoxia-inducible factor (HIF1A and HIF2A), angiogenesis, and chemoradiotherapy outcome of squamous cell headand-neck cancer. Int J Radiat Oncol Biol Phys 2002; 53(5): 1192-1202.

11 Dix TA, Aikens J. Mechanisms and biological relevance of lipid peroxidation initiation. Chem Res Toxicol 1993; 6(1): 2-18.

12 Kourie JI. Interaction of reactive oxygen species with ion transport mechanisms. Am J Physiol 1998; 275(1): 1-24

13 McConnell EJ, Bittelmeyer AM, Raess BU. Irreversible inhibition of plasma membrane $\left(\mathrm{Ca}^{2+}+\mathrm{Mg}^{2+}\right)$-ATPase and $\mathrm{Ca}^{2+}$ transport by 4-OH-2,3-trans-nonenal. Arch Biochem Biophys 1999; 361(2): 252-256.

14 Tyurina YY, Shvedova AA, Kawai $\mathrm{K}$ et al. Phospholipid signaling in apoptosis: peroxidation and externalization of phosphatidylserine. Toxicology 2000; 148(2/3): 93-101.

15 Hanafy KA, Krumenacker JS, Murad F. NO, nitrotyrosine, and cyclic GMP in signa transduction. Med Sci Monit 2001; 7(4): 801-819.

16 Hughes MN. Relationships between nitric oxide, nitroxyl ion, nitrosonium cation and peroxynitrite. Biochim Biophys Acta 1999; 1411(2/3): 263-272.

17 American Joint Committee on Cancer. Manual for Staging of Cancer. 4th ed Philadelphia: JB Lippincott, 1992.

18 Jablonska E, Kiersnowska-Rogowska B, Ratajczak W et al. Reactive oxygen and nitrogen species in the course of B-CLL. Adv Med Sci 2007; 52(1): 154-158.

19 Singh S, Gupta AK. Nitric oxide: role in tumour biology and iNOS/NO-based anticancer therapies. Cancer Chemother Pharmacol 2011; 67(6): 1211-1224.
20 Wink DA, Mitchell JB. Chemical biology of nitric oxide: insights into regulatory, cytotoxic, and cytoprotective mechanisms of nitric oxide. Free Radic Biol Med 1998; 25(4/5): 434-456.

21 Beevi SS, Rasheed AM, Geetha A. Evaluation of oxidative stress and nitric oxide levels in patients with oral cavity cancer. Jpn J Clin Oncol 2004; 34(7): 379-385.

22 Rasheed $\mathrm{MH}$, Beevi SS, Geetha A. Enhanced lipid peroxidation and nitric oxide products with deranged antioxidant status in patients with head and neck squamous cell carcinoma. Oral Oncol 2007; 43(4): 333-338.

23 Gokul S, Patil VS, Jailkhani $\mathrm{R}$ et al. Oxidant-antioxidant status in blood and tumor tissue of oral squamous cell carcinoma patients. Oral Dis 2010; 16(1): 29-33.

24 Jablonska E, Puzewska W, Charkiewicz M. Effect of IL-18 on leukocyte expression of iNOS and phospho-IkappaB in patients with squamous cell carcinoma of the oral cavity. Neoplasma 2006; 53(3): 200-205.

25 Gavilanes J, Moro MA, Lizasoain I et al. Nitric oxide synthase activity in human squamous cell carcinoma of the head and neck. Laryngoscope 1999; 109(1): 148152.

26 Brennan PA, Thomas GJ, Langdon JD. The role of nitric oxide in oral diseases. Arch Oral Biol 2003; 48(2): 93-100.

27 Connelly ST, Macabeo-Ong M, Dekker $\mathrm{N}$ et al. Increased nitric oxide levels and iNOS over-expression in oral squamous cell carcinoma. Oral Oncol 2005; 41(3): 261-267.

28 Thomsen LL, Scott JM, Topley P et al. Selective inhibition of inducible nitric oxide synthase inhibits tumor growth in vivo: studies with $1400 \mathrm{w}$, a novel inhibitor. Cancer Res 1997; 57(1): 3300-3304.

29 Ambs S, Hussain SP, Harris CC. Interactive effects of nitric oxide and the p53 tumor suppressor gene in carcinogenesis and tumor progression. FASEB J 1997; 11(6): 443-448.

30 Gallo 0 , Masini E, Morbidelli $\mathrm{L}$ et al. Role of nitric oxide in angiogenesis and tumor progression in head and neck cancer. J Nat/ Cancer Inst 1998; 90(8): 587-596.

31 Morbidelli L, Chang $\mathrm{CH}$, Douglas JG et al. Nitric oxide mediates mitogenic effect of VEGF on coronary venular endothelium. Am J Physiol 1996; 170(1 Pt 2): 411-415.

32 Kawamata $\mathrm{H}$, Uchida D, Hamano $\mathrm{H}$ et al. Active-MMP2 in cancer cell nests of ora cancer patients: correlation with lymph node metastasis. Int J Oncol 1998; 13(4): 699-704.

33 Kurahara S, Shinohara M, Ikebe T et al. Expression of MMPS, MT-MMP, and TIMPs in squamous cell carcinoma of the oral cavity: correlations with tumor invasion and metastasis. Head Neck 1999; 21(7): 627-638.

34 Maeda H, Noguchi Y, Sato K et al. Enhanced vascular permeability in solid tumor is mediated by nitric oxide and inhibited by both new nitric oxide scavenger and nitric oxide synthase inhibitor. Jpn J Cancer Res 1994; 85(4): 331-334.

35 Przybyszewski W, Kasperczyk J, Stoklosa K et al. [DNA damage induced by products of lipid peroxidation.] Post Hig Med Dosw 2005; 59(17): 75-81. Polish.

36 Torun M, Yardim S, Gonenc A et al. Serum beta-carotene, vitamin E, vitamin C and malondialdehyde levels in several types of cancer. J Clin Pharm Ther 1995; 20(5): 259-263.

37 Sabitha KE, Shyamaladevi CS. Oxidant and antioxidant activity changes in patients with oral cancer and treated with radiotherapy. Oral Oncol 1999; 35(3): 273-277.

38 Korde SD, Basak A, Chaudhary M et al. Enhanced nitrosative and oxidative stress with decreased total antioxidant capacity in patients with oral precancer and oral squamous cell carcinoma. Oncology 2011; 80(5/6): 382-389.

39 Broncel M, Balcerak M, Bala A et al. [The erythrocyte membrane structure in patients with mixed hyperlipidemia.] Wiad Lek 2007: 60(1/2): 4-9. Polish.

40 Yeo WS, Lee SJ, Lee JR et al. Nitrosative protein tyrosine modifications: biochemistry and functional significance. J Biochem Mol Biol 2008; 41(3): 194-203.

41 Mantovani A, Allavena P, Sica A et al. Cancer-related inflammation. Nature 2008; 454(7221): 436-444.

This work is licensed under a Creative Commons

Attribution-NonCommercial-NoDerivative Works 3.0

Unported License. To view a copy of this license, visit http:// creativecommons.org/licenses/by-nc-nd/3.0 